\title{
Ley Nacional de Fertilización Asistida: avances y desafíos
}

\author{
National Law on Assisted Reproduction: Advancements and Challenges
}

Romina Pesce* y Gastón Perman**

\begin{abstract}
Resumen
Recientemente fue sancionada en la Argentina la Ley Nacional 26.862 sobre fertilización asistida, la primera en su tipo en Latinoamérica. Este artículo aborda los avances que la misma representa para los beneficiarios, así como los desafíos que deben ser tenidos en cuenta a la hora de su reglamentación e implementación desde la perspectiva del sistema de salud y de la sociedad en general. Creemos que sólo con una visión amplia y objetiva que contemple estos aspectos y dimensiones se podrá potenciar su impacto positivo y minimizar sus consecuencias negativas.
\end{abstract}

\section{Abstract}

Argentina recently passed the National Law 26,862 on assisted reproduction, the first one of its kind in Latin America. This paper deals with the benefits for the potential users, as well as the challenges that should be taken into account from the perspective of the health care system and the society in general. We believe that its positive impact could be maximized and its negative consequences minimized only through a comprehensive and objective analysis that consider these aspects and dimensions.

Palabras clave: legislación, Etica, Justicia Social, Infertilidad, Técnicas reproductivas. Key words: elegislation, Ethics, Social justice, Infertility, Reproductive Techniques.

Pesce R y Perman G. Ley Nacional de Fertilización Asistida: avances y desafíos. Evid Act Pract Ambul Abr-Jun 2013(16),2:42-44

Introducción

Recientemente, y luego de una larga espera para muchos, fue sancionada la Ley Nacional 26.862, de acceso integral a los procedimientos y técnicas médico-asistenciales de reproducción médicamente asistida'. La misma representa un gran avance en muchos sentidos. Es la primera ley nacional en Latinoamérica que, teniendo en cuenta que la infertilidad es un problema de salud ${ }^{2}$, asegura el derecho a recibir tratamiento para la misma. Dicha ley fue votada por una amplia mayoría de los legisladores y, en general, fue bien recibida por buena parte de la sociedad y los medios de comunicación ${ }^{3}$. Sin embargo, creemos que las implicancias y alcances de esta ley no fueron lo suficientemente debatidos $\mathrm{o}$, al menos, los comentarios se concentraron en sólo algunos aspectos, más relacionados con intereses sectoriales.

Este artículo se escribió luego de ser aprobada la ley pero antes de ser reglamentada. El objetivo del mismo es aportar a la discusión sobre las consideraciones necesarias a la hora de reglamentar e implementar la ley para optimizar sus resultados e impacto positivo y limitar sus potenciales consecuencias negativas. Para intentar una mayor claridad, se realizará un análisis de las posibles implicancias de la ley en tres dimensiones o perspectivas: la de las personas beneficiarias de la misma, la del sistema de salud, y la de la sociedad general.

\section{Dimensión de los beneficiarios de la ley}

La Ley Nacional 26.862, de acceso integral a los procedimientos y técnicas médico-asistenciales de reproducción médicamente asistida es amplia e inclusiva. No discrimina por edad, estado civil u orientación sexual. Promueve la accesibilidad para todos al tratamiento integral de la infertilidad (el único requisito que dispone la ley es que se haya explicitado el consentimiento informado del o de los beneficiarios). Esto implica no sólo la cobertura de las técnicas de reproducción asistida (por ejemplo, fertilización in vitro), sino también todo lo relacionado con el tratamiento médico y/o psicológico de las personas que así lo requieran. Esto constituye un gran avance respecto a la Ley 14.208 de la Provincia de Buenos Aires, aprobada a fines de 2010. Dicha ley, la primera a nivel provincial en legislar sobre este tema en la Argentina, es mucho más restrictiva ${ }^{4}$. Limita, por ejemplo, el acceso a este tipo de tratamientos para parejas (no considera la paternidad monoparental) y con espermatozoides y óvulos (ovocitos) propios. Esto se denomina téc- nicamente fertilización homóloga, y excluye la donación de dichas células (gametas) por otra/s persona/s ajena/s a la pareja). Su decreto reglamentario, 2.980 de 2011, aclara que la mujer debe tener entre 30 y 40 años de edad 5 . Por lo tanto, es indiscutible que desde la perspectiva de los beneficiarios, la Ley Nacional 26.862 no excluye a nadie que tenga deseos actuales de procreación.

Esta nueva Ley Nacional no se queda sólo en la búsqueda actual de embarazo sino que también garantiza el acceso a técnicas de preservación de la fertilidad "para aquellas personas, incluso menores de dieciocho (18) años que, aun no queriendo llevar adelante la inmediata consecución de un embarazo, por problemas de salud o por tratamientos médicos o intervenciones quirúrgicas puedan ver comprometidas su capacidad de procrear en el futuro"1. En este sentido, la ley puede contribuir a la preservación efectiva de la fertilidad en esta población vulnerable, no sólo mejorando el acceso a estos servicios desde la cobertura financiera, sino también promoviendo el conocimiento de estas técnicas entre la comunidad médica. Este mayor conocimiento de la existencia de técnicas novedosas (pero poco indicadas o utilizadas) para preservar la capacidad reproductiva actuaría aumentando la indicación médica de las mismas en este grupo de riesgo, beneficiándolos claramente ${ }^{6}$.

Además, la ley no se limita a las técnicas actuales sino que considera también explícitamente la inclusión de "nuevos procedimientos y técnicas desarrollados mediante avances técnico-científicos, cuando sean autorizados por la autoridad de aplicación" 1

Por lo tanto, desde la perspectiva de los beneficiarios y al menos en lo que respecta al texto de la ley en sí -previamente a su reglamentación-, la misma es amplia e inclusiva, y además considera potenciales problemas y posibles soluciones (actuales o futuras) que pueda tener cualquier persona en relación con su deseo de reproducción.

\section{Dimensión del Sistema de Salud}

Analizada en la dimensión del sistema de salud como un todo, la ley representa un gran desafío en cuanto asegurar una buena cobertura de estos servicios de fertilización asistida por un lado, y la asignación de recursos escasos y la priorización de necesidades sanitarias en general por el otro?. La ley dispone

* Servicio de Ginecología del Hospital Italiano de Buenos. romina.pesce@ @iba.org.ar

** Departamento de Salud Pública del Instituto Universitario Hospital Italiano y Servicio de Clínica Médica del Hospital Italiano de Buenos Aires. 
que tanto el sector público, como el de las obras sociales y las entidades de medicina prepaga y "...todos aquellos agentes que brinden servicios médico-asistenciales a sus afiliados, independientemente de la figura jurídica que posean, incorporarán como prestaciones obligatorias y a brindar a sus afiliados o beneficiarios, la cobertura integral e interdisciplinaria del abordaje, el diagnóstico, los medicamentos y las terapias de apoyo y los procedimientos y las técnicas que la Organización Mundial de la Salud (OMS) define como reproducción médicamente asistida (...) Quedan incluidos en el Programa Médico Obligatorio (PMO) (...) con los criterios y modalidades de cobertura que establezca la autoridad de aplicación". Para ello, en su artículo noveno dispone que "... a los fines de garantizar el cumplimiento de los objetivos de la presente ley el Ministerio de Salud de la Nación deberá proveer anualmente la consiguiente asignación presupuestaria"1.

Hasta aquí tenemos entonces una ley concebida con las mejores intenciones y con mandato explícito para una asignación presupuestaria específica y la cobertura integral en reproducción asistida por parte del PMO. Sin embargo, dadas las actuales limitaciones de nuestro sistema de salud, le pregunta es cómo se logrará esto en un sistema fragmentado, desfinanciado e ineficiente $e^{8-10}$.

Si tenemos en cuenta que hay una relación estrecha entre la accesibilidad, la calidad y los costos en salud, en donde si un factor se modifica, necesariamente se modificará al menos uno de los otros dos, podremos deducir lo siguiente: al aumentar el acceso a servicios de reproducción asistida (el objetivo buscado), van a aumentar los costos y/o a disminuir la calidad de atención (en estos servicios y/o en otros brindados habitualmente). Algunos podrán o intentarán trasladar parte de esos costos a los usuarios (por ej. empresas de medicina prepaga), aumentando la cuota a todos los afiliados, dificultando el acceso general a los servicios de salud. Pero los que no puedan hacer esto, deberán reasignar recursos que estaban previamente destinados a otros servicios. Si estos servicios eran superfluos o innecesarios, perfecto. Pero si no, podrían resentirse la oferta (acceso) y/o calidad de dichos servicios o productos (por ejemplo cirugías programadas, prótesis, vacunación, etc.).

También se debe considerar que las técnicas de reproducción asistida se asocian a mayor incidencia de embarazos de alto riesgo, partos pretérmino e internaciones en neonatología ${ }^{11}$; y que estos costos también deben ser sumados a los de los servicios de reproducción asistida cuando analizamos el problema desde la perspectiva del sistema de salud.

Respecto a esta relación entre acceso, costos y calidad, es interesante analizar lo que sucedió con la reproducción asistida en la Provincia de Buenos Aires. En Diciembre de 2010 se sancionó la Ley Provincial 14.208. Como mencionamos previamente, la misma es más restrictiva que la ley nacional. Cubre sólo a parejas y únicamente técnicas de fertilización homóloga reconocidas por la $\mathrm{OMS}^{4}$. Recordemos también que el decreto 2.980 que reglamenta la ley restringe todavía más la cobertura. Por ejemplo, cubre sólo a mujeres entre 30 y 40 años de edad, dando prioridad a las parejas que no tengan hijos producto de dicha relación ${ }^{5}$. Algunos podrán decir que esto es injusto, quizás con razón. Otros, que es más pragmática o realista, reconociendo implícitamente la escasez de recursos y priorizando la población que más se pueda beneficiar de servicios de reproducción asistida. Esto es discutible, por supuesto, pero vale destacar que aún varios países desarrollados con mucho más presupuesto en salud también tienen rangos de edad de elegibi-lidad en la mujer ${ }^{12}$. Vemos aquí algún intento de racionalizar (no sólo racionar) el gasto en función de optimizar el beneficio. Probablemente ambas visiones tengan razón en parte. Pero es seguro que la discusión debe girar en torno a cómo ofrecer un servicio con un adecuado balance entre accesibilidad, calidad y costos, que sea viable y sustentable en el tiempo.

Siguiendo el caso de la Provincia de Buenos Aires, cuando analizamos la resolución de IOMA respecto a la cobertura en fertilización asistida, vemos que las restricciones vuelven a aumentar. Entre ellas se puede mencionar que sólo cubre dos (máximo tres) ciclos de alta complejidad en total, y sólo uno por año, y no incluye la medicación para la estimulación ovárica ${ }^{13}$. Aun con estas formas de contener costos (a expensas de disminuir el acceso a otros posibles beneficiarios que no cumplan con los criterios establecidos, y de limitar la cobertura de servicios y/o insumos), existen actualmente en la Provincia de Buenos Aires múltiples barreras para el acceso a estas técnicas de reproducción asistida incluso para las personas elegibles $^{14}$. A su vez, esto genera una judicialización del problema a través de amparos para solicitar la cobertura de estos servicios (incluso los no cubiertos). Esto trae otro tipo de problemas que serán analizados más adelante. La ley nacional, si bien plantea una cobertura amplia buscando evitar esto, sólo llevará a una mayor judicialización si no se le brinda a los financiadores (Estado, Obras Sociales o prepagas) una fuente de financiación genuina para hacerse cargo de los nuevos costos en salud generados.

Por otro lado, la ley nacional puede tener efectos positivos debido a varios aspectos que considera explícitamente: la formación y capacitación continua de recursos humanos; el registro único de instituciones autorizadas, lo que puede facilitar la supervisión y favorecer la mejora de procesos; la publicación de la lista de los centros de referencia para mejorar el conocimiento de la población sobre los centros habilitados y mejorar el acceso (también podría favorecer la transparencia y la mejora de la calidad por competencia entre las instituciones prestadoras por los usuarios mejor informados); y campañas de información para promover los cuidados de la fertilidad.

Sin embargo, esto también puede estar reñido con la forma en la que se reglamente la ley o las resoluciones de los financiadores respecto a la cobertura efectiva que se terminará dando. Por ejemplo, IOMA sólo cubre determinados centros prestadores. Eso no necesariamente es malo, pero podría, por ejemplo, limitar los beneficios de la libre competencia entre instituciones por captar a los usuarios como se planteó previamente.

En síntesis, en un país donde existen antecedentes de falta de reglamentación de leyes ${ }^{15} \mathrm{o}$ donde dicha reglamentación termina restringiendo la finalidad que persigue la ley, los resultados finales pueden ser muy diversos. Lo que queda claro es que la interrelación entre acceso, calidad y costos no puede soslayarse. Sin asegurar genuinamente la forma de financiar el mayor acceso a estos servicios de salud, el sistema terminará regulándose "solo" en detrimento de al menos uno de estos pilares.

\section{Dimensión de la sociedad}

Una ley como esta que estamos analizando, que otorga derechos con un espíritu inclusivo, va de la mano de una sociedad más abierta y tolerante. Es cierto que esto sólo no lo asegura, pero es un avance en esa dirección. Ahora bien, aun cuando la ley demoró mucho tiempo en ser aprobada, hubo un debate entre algunos de los actores sociales involucrados, pero no 
entre todos los que podrían sentirse afectados directa o indirectamente, que habría contribuido a generar un consenso social. Pero aun así, la ley fue aprobada por amplia mayoría y fue bien recibida por la prensa en general ${ }^{3}$. Por lo tanto, puede funcionar como un punto de partida sobre el cual, con el tiempo, se termine generando una mayor aceptación social de hecho respecto de algunos temas controvertidos. Durante este proceso, sin embargo, podrían generarse consecuencias negativas para algunos como discriminación y/o estigmatización. Es algo muy complejo con profundas raíces sociales, culturales y religiosas. Probablemente estas consecuencias sean inevitables, dado que siempre que se producen cambios importantes en el "statu quo" de una sociedad surgen tensiones y conflictos. La ley nacional menciona que deben realizarse campañas de información' ${ }^{1}$. Si bien detalla que el fin es promover los cuidados de la fertilidad, si se utilizaran en parte también para favorecer este consenso social y la aceptación de familias diferentes a la "tradicional", dichos conflictos podrían disminuirse.

Un aspecto que no le compete directamente a esta ley pero que debe ser tenido en cuenta es el hueco legal que existe actualmente respecto a la maternidad en los casos de útero subrogado. Al aumentar los casos, la ley podría favorecer los reclamos judiciales para lograr el reconocimiento de la paternidad. Sin embargo, acaba de darse un fallo favorable en la justicia para una pareja que ha tenido una hija en un útero "prestado"16. Además, el nuevo Código Civil contemplaría estos casos, por lo que a futuro este no sería un problema legal tan importante. Permanecen sí las consideraciones y desafíos éticos asociados a esta técnica de reproducción asistida ${ }^{17}$. Lo mismo puede decirse de los derechos del niño por nacer, que no fueron abordados explícitamente.

Quizás uno de los temas más importantes para analizar es el de la equidad. Vimos que desde la perspectiva de los beneficiarios directos, estaría generando mayor equidad. Según el texto de la ley, todo aquel que necesite servicios relacionados con fertilización asistida podría acceder al mismo, sin distinción de edad, estado civil u orientación sexual. Pero desde la dimensión del sistema de salud y de la sociedad en general, el efecto podría ser el opuesto. Grupos con mayor capacidad de presión (mayor conocimiento, nivel educativo, económico, etc.), o con acceso a obras sociales o prepagas que puedan dar respuesta a mayores demandas, o que tengan más facilidades para solicitar amparos judiciales, podrían tener ahora mayor acceso a los servicios de fertilización asistida que los grupos con menos recursos o posibilidades. Esto es algo que puede pasar con cualquier servicio de salud, no es problema de la ley nacional de fertilidad per se. El problema puede surgir por la forma en la que se implemente. Si lo que establece la ley no es acompañado por un aumento de recursos y posibilidades de acceso a servicios de calidad para los grupos más desfavorecidos en una forma proporcionalmente igual o mayor que para los grupos favorecidos, podría aumentar la inequidad. Esta inequidad contribuiría finalmente a tener un sistema de salud y una sociedad menos justos.

\section{Conclusión}

La Ley Nacional 26.862 es inclusiva, amplía derechos y en su texto busca favorecer la accesibilidad integral a servicios de reproducción asistida. Tiene un espíritu moderno y progresista, pudiendo generar cambios importantes en la conformación de la sociedad y en sus valores y dinámica.

Sin embargo, existen desafíos importantes. Más allá de los vacíos legales que exceden los alcances de esta ley, los desafíos están fundamentalmente relacionados con la forma en la que se la termine reglamentando e implementando. Si se asegura una fuente genuina que cubra los nuevos costos para el sistema de salud, sin quitarle recursos a otros temas prioritarios, tanto sanitarios como sociales; si se disponen las medidas necesarias para favorecer un acceso equitativo y de buena calidad; y si realmente se busca generar un consenso social y una aceptación de nuevos tipos de familia, vínculos y valores, entonces la ley va a ver respetado su espíritu y la finalidad que persigue.

Este artículo busca colaborar con la discusión del tema en forma amplia y objetiva y favorecer el debate necesario para potenciar el impacto positivo de la ley y minimizar consecuencias negativas. Ese es el desafío.

Recibido el 12/07/13 y aceptado el 22/07/13.

\footnotetext{
Referencias

1. Congreso de la Nación Argentina. Ley Nacional 26.862. Acceso integral a los procedimientos y técnicas médico-asistenciales de reproducción médicamente asistida. 2013

2. ICMART, WHO. The International Committee for Monitoring Assisted Reproductive Technology (ICMART) and the World Health Organization (WHO) Revised Glossary on ART Terminology, 2009. Human Reproduction. 2009;24(11):2683-7. 4. Ley Provincial 14.208. Provincia de Buenos Aires; 2010

6. Pesce R, Gogorza S. Preservación de la fertilidad en pacientes adolescentes y mujeres jóveneses. In: Asociación Latinoamericana de Endocrinología Ginecológica (ALEG), editor. Endocrinología ginecológica. Una visión latinoamericana en el siglo XXI. Buenos Aires; 2013. p. 157-69.

7. Organización Mundial de la Salud. Respuesta a las necesidades sanitarias urgentes. Informe sobre la salud en el mundo 2006. Ginebra; 2006. Capítulo 2.

8. Vilosio J. Las promesas incumplidas de la Salud en Argentina (primera parte). Evid. actual. práct. ambul. 2008;11(4):98-100.

9. Vilosio J. Las promesas incumplidas de la Salud en Argentina (segunda parte). Evid. actual. práct. ambul. 2010;13(2):42-4.

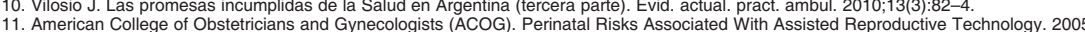

12. National Institute for Health and Clinical Excellence (NICE). Fertility. Assessment and treatment for people with fertility problems. 2013.

13. Instituto Obra Médico Asistencial (IOMA). Resolución 8538/2010. Cobertura de Fertilización Asistida por parte del IOMA. 2010.

14. Concebir (Grupo de Apoyo para personas con Trastornos en la Reproducción). El amparo como camino [Internet]. 2013 [accedido el 23 Jun 2013]. Disponible en: http://www.concebir.org.ar/core/?q=content/elamparo-como-camino

15. Cerutti JC. Leyes que no terminan de reglamentarse. La Nación. 2013 Jun 30;10 / Empleos.

16. Gioberchio G. La Justicia inscribió a una nena gestada en un vientre prestado. Clarín. 2013 Jun 26;30-
} 